\title{
Recognition of our outstanding reviewers
}

Published online: 16 November 2013

(C) Am Soc Emergency Radiol 2013

The following list names the experts who have volunteered their time and knowledge to review manuscripts for Emergency Radiology in the past year. Without their work and expertise publication of this journal would not be possible. On behalf of the Editorial Board and our readers, I thank these reviewers.

Afaq, Asim

Aghaei-Lasboo, Anahita

Asrani, Ashwin

Aygun, Nafi

Baker, Stephen

Barron, Dominic

Bennett, D Lee

Berger, Ferco

Bilow, Ronald

Bisker, Jeffrey

Blackmore, Christopher

Bui-Mansfield, Liem

Buls, Nico

Camera, Luigi

Carrafiello, Gianpaolo

Catalano, Orlando

Chong, Suzanne

Chung, Jonathan

Coughlin, Bret

Courcoutsakis, Nikos

Danza, Francesco

Dhand, Sabeen

Ding, Alexander

Doris, Peter

Doss, Arockia

Duran-Mendicuti, Alejandra

Feletti, Francesco

Filippi, Christopher

Geijer, Mats

Gerstenmaier, Jan
Gibbs, Robert

Goldman, Stanford

Gulati, Kavita

Gunn, Martin

Hardie, Andrew

Hatem, Stephen

Heller, Matthew

Hemmati, Masoud

Herliczek, Thaddeus

Horowitz, Jeanne

Inamasu, Joji

Jae, Hwan Jun

John, Susan

Johnson, Jamlik-Omari

Kalina, Peter

Kalyanpur, Arjun

Kamper, Lars

Katsanos, Konstantinos

Katz, Douglas S.

Kilickesmez, Ozgur

Kocakoc, Ercan

Kool, Digna

Kose, Ozkan

Krokidis, Miltiadis

Kumar, Atin

Landman, Wendy

Lanzman, Rotem

Lee, Wai-Kit

Lemos, Alessandro

Levenson, Robin
Ronald J. Zagoria, MD

Editor-in-Chief, Emergency Radiology

Lewiss, Resa

Liaw, Voon

Lien, Li-Chang

Lim, Tze Chwan

Linnau, Ken

Lucey, Brian

Maizlin, Zeev

Maldonado, Jose

Maurea, Simone

Mehta, Kaushal

Mennitt, Kevin

Mian, Asim

Mirvis, Stuart

Monu, Johnny

Morgan, John

Nath, Jaya

Nunez, Diego

Pellã ${ }^{3} \mathrm{n}, \operatorname{RaA}{ }^{\circ} l$

Pierre-Jerome, Claude

Puig, Stefan

Reddy, Sravanthi

Riascos, Roy

Robinson, Jeffrey

Rocha, Tatiana

Sanchez, Thomas Ray

Scaglione, Mariano

Sefczek, Robert

Shriki, Jabi

Sliker, Clint

Small, William 
Sodickson, Aaron

Soto, Jorge

Steenburg, Scott

Strub, William

Sunnapwar, Abhijit

Swischuk, Leonard

Symons, Sean

Tajima, Hiroyuki

Theodorou, Daphne

Tonerini, Michele

Tosun, Ozgur
Tuntiyatorn, Lojana

Turk, Figen

Turkbey, Baris

Udayasankar, Unni

Ulusan, Serife

Uysal, Ender

van Kuijk, C.

van Rijn, Rick

Victoria, Teresa

Vollmer, Ivan
Webb, Emily

Wong, Yon-Cheong

Yarmohammadi, Hooman

Yildiz, Sema

Yu, Joseph

Zacharia, T. Thomas

Zaragoza, Edward 